Supporting Information:

\title{
The CU Airborne Solar Occultation Flux Instrument: Performance evaluation during BB-FLUX
}

Natalie Kille ${ }^{1,2,3, a}$, Kyle J. Zarzana ${ }^{1}$, Johana Romero Alvarez ${ }^{1}$, Christopher F. Lee ${ }^{1,2}$, Jake P. Rowe ${ }^{1,2}$, Benjamin Howard ${ }^{1}$, Teresa Campos ${ }^{4}$, Alan Hills ${ }^{4}$, Rebecca S. Hornbrook ${ }^{4}$, Ivan Ortega ${ }^{4}$, Wade Permar ${ }^{5}$, I Ting Ku ${ }^{6}$, Jakob Lindaas ${ }^{6}$, Ilana B. Pollack ${ }^{6}$, Amy P. Sullivan ${ }^{6}$, Yong Zhou ${ }^{6}$, Carley D. Fredrickson ${ }^{7}$, Brett B. Palm ${ }^{7, b}$, Qiaoyun Peng ${ }^{7}$, Eric C. Apel ${ }^{4}$, Lu Hu ${ }^{5}$, Jeffrey L. Collett Jr. ${ }^{6}$, Emily V. Fischer ${ }^{6}$, Frank Flocke ${ }^{4}$, James W. Hannigan ${ }^{4}$, Joel Thornton ${ }^{7}$, and Rainer Volkamer ${ }^{1,2,3, *}$

${ }^{1}$ Department of Chemistry, University of Colorado Boulder, Boulder, Colorado 80309, USA

${ }^{2}$ Cooperative Institute for Research in Environmental Sciences, University of Colorado Boulder, Boulder, Colorado 80309, USA

${ }^{3}$ Department of Atmospheric and Oceanic Sciences, University of Colorado Boulder, Boulder, Colorado 80309, USA

${ }^{4}$ Atmospheric Chemistry Observations \& Modeling, National Center for Atmospheric Research, Boulder, Colorado 80301, USA

${ }^{5}$ Department of Chemistry and Biochemistry, University of Montana, Missoula, Montana 59812, USA

${ }^{6}$ Department of Atmospheric Science, Colorado State University, Fort Collins, Colorado 80521, USA

${ }^{7}$ Department of Atmospheric Sciences, University of Washington, Seattle, Washington 98195, USA

a Now at: Institute of Energy and Climate Research: Troposphere (IEK-8), Forschungszentrum Jülich $\mathrm{GmbH}, 52425$ Jülich, Germany

${ }^{b}$ Now at: Atmospheric Chemistry Observations \& Modeling, National Center for Atmospheric Research, Boulder, Colorado 80309, USA

* Corresponding author: Rainer Volkamer (rainer.volkamer@colorado.edu)

The Supporting information file includes a brief description of the retrieval settings used for the NCAR HR-FTS data analysis, as well as 7 Supplement Figures, 3 Supplement Tables, and 3 references.

For all gases retrieved by the NCAR HR-FTS the recommendations of the Network for Detection of Atmospheric Composition Change InfraRed Working Group (NDACC IRWG; www2.acom.ucar.edu/irwg) were applied. A larger than usual enhancement in the $\mathrm{C}_{2} \mathrm{H}_{6}$ VCD on 9 November 2018 caused elevated residual structures due to under-accounted pressure broadening in the high-resolution spectra; these residuals were caused by under-accounted $\mathrm{C}_{2} \mathrm{H}_{6}$ absorption (pressure broadening from low altitude $\mathrm{C}_{2} \mathrm{H}_{6}$ plumes) and accompanied by lower $\mathrm{C}_{2} \mathrm{H}_{6}$ VCDs compared to those measured by CU AirSOF. An increase in the diagonal of the covariance matrix $\left(\mathrm{S}_{\mathrm{a}}\right)$ of $\mathrm{C}_{2} \mathrm{H}_{6}$ by a factor 1.75 reduced these residuals, and was necessary to provide flexibility to the retrieval to capture the large $\mathrm{C}_{2} \mathrm{H}_{6}$ VCD enhancements on that day. 


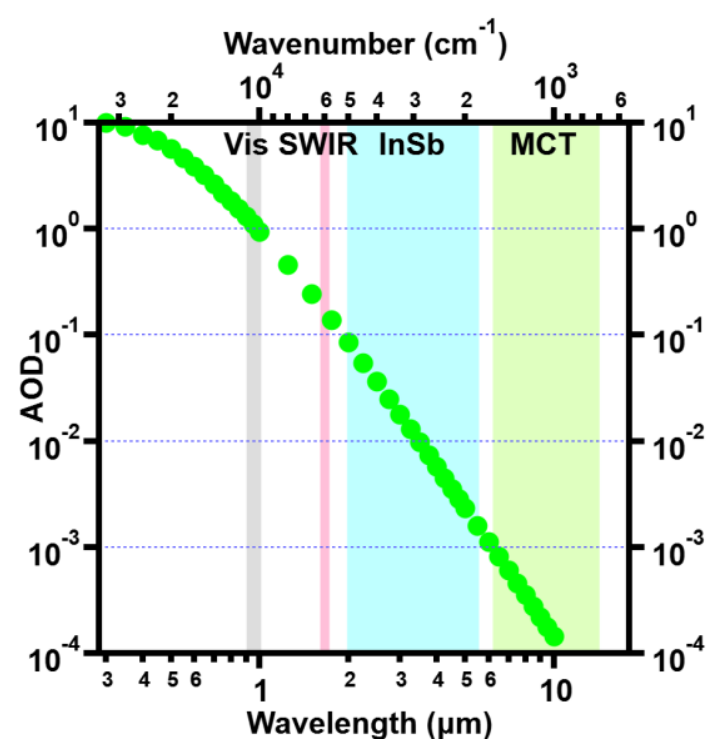

Figure S1: Changes in AOD (for a constant refractive index of $m=1.6+0 i$ ) as a function of wavelength/ wavenumber covering the CU AirSOF instrument components: Vis and SWIR indicate the operating range of the cameras of the dual camera design, InSb and MCT are the two detectors of the EM27 FTS. 


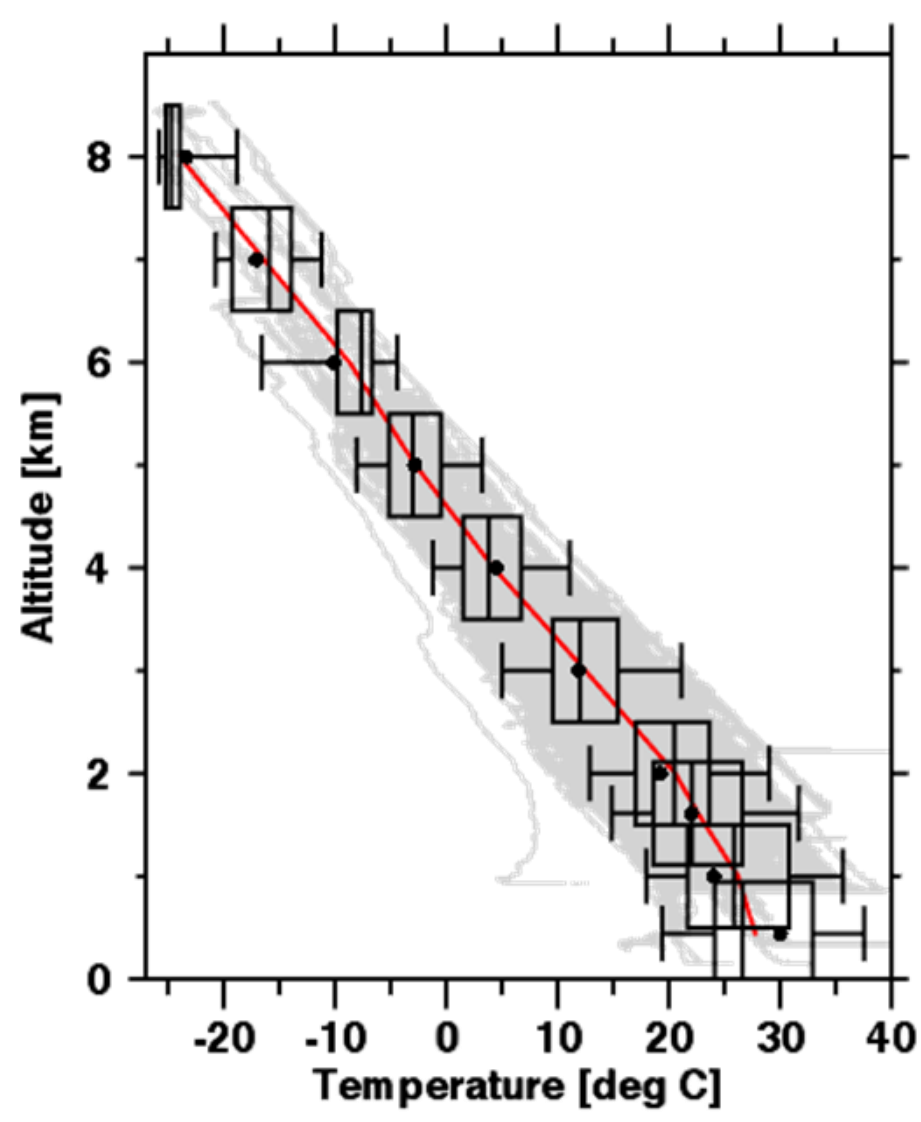

Figure S2: Observed temperatures during BB-FLUX. Gray data points represent individual measurements. Black data points are the temperature values used in the a priori information for SFIT. The box and whiskers $\left(5^{\text {th }}, 25^{\text {th }}, 50^{\text {th }}, 75^{\text {th }}, 95^{\text {th }}\right.$ percentile) and the average (red line) are calculated in $1 \mathrm{~km}$ altitude bins around the altitude layers of the SFIT a priori based on the measurements. The difference between the temperature used in the retrieval and the measurements is $\sim 1 \mathrm{~K}$ when comparing to the $50^{\text {th }}$ percentile, $\sim 3 \mathrm{~K}$ when comparing to the $25^{\text {th }}$ or $75^{\text {th }}$ percentile, and $\sim 7 \mathrm{~K}$ when comparing to the $5^{\text {th }}$ or $95^{\text {th }}$ percentile. The temperature component during the SFIT error budget analysis was set at $10 \mathrm{~K}$ uncertainty for all altitudes of the a priori. 


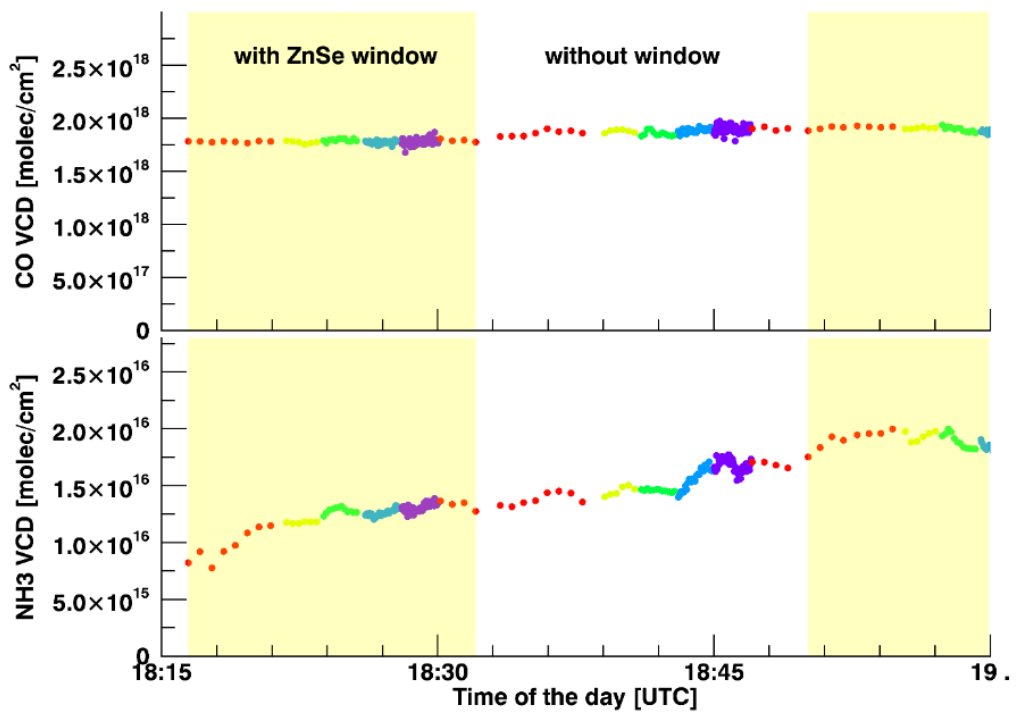

Figure S3: Exemplary timeseries of $\mathrm{CO}$ (InSb detector) and $\mathrm{NH}_{3}$ (MCT detector) measured on 7 June 2018 on the Volkamer roof-top lab in Boulder, Colorado, with and without solar tracking through the ZnSe window. Different colors indicate varying numbers of scans per spectrum acquisition. The ZnSe window transmission near unity results in smooth transition (captured within 2 standard deviations of the 5-min period prior and post transition as well as the total VCD error) of the measurements as the atmosphere changes over time. 


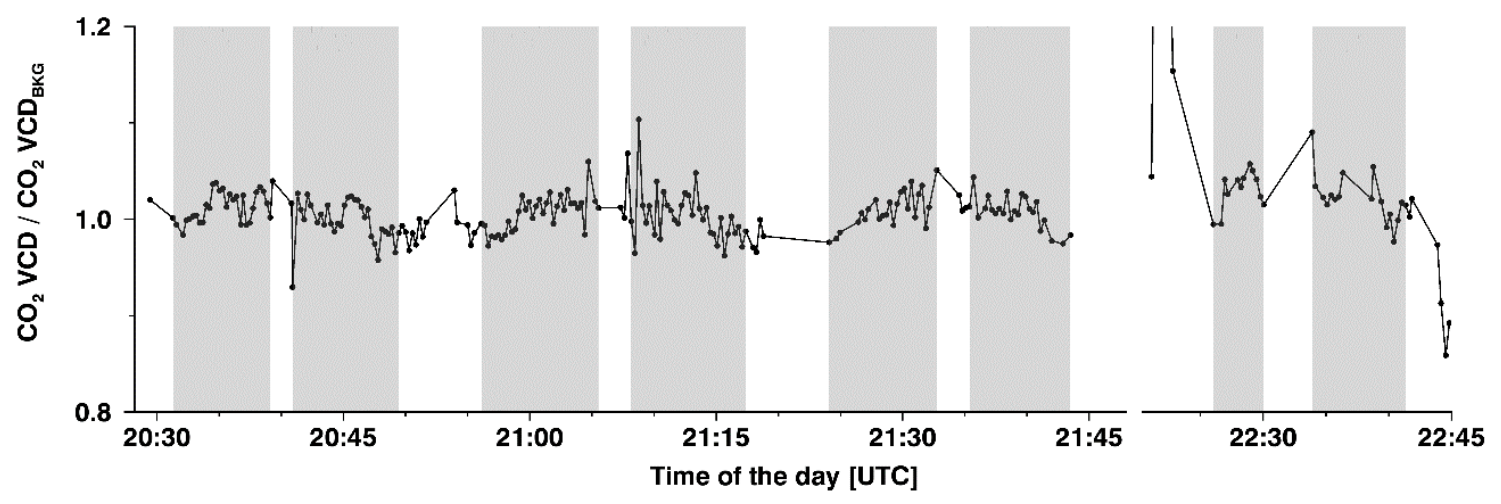

Figure S4: Change in $\mathrm{CO}_{2}$ measured by $\mathrm{CU}$ AirSOF (shown from interfering species in $\mathrm{NH}_{3}$ retrieval) while transitioning between background air and passing underneath the Rabbit Foot Fire plume, indicated by the shaded periods, on 15 August 2018. 


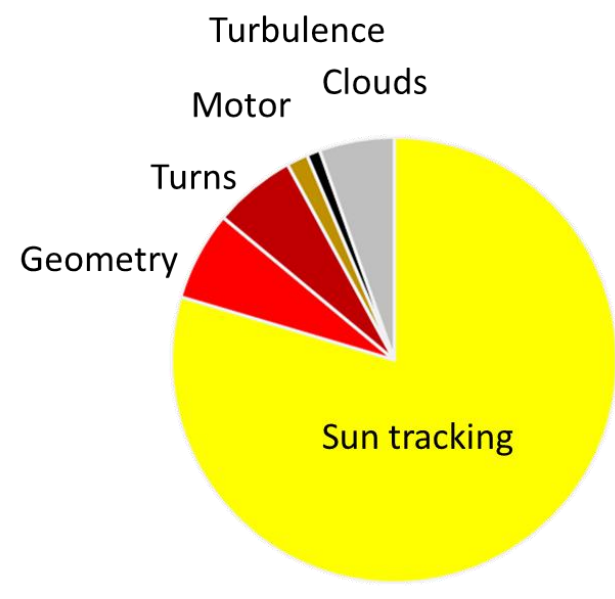

Figure S5: Solar tracking success and limitations during the BB-FLUX deployment.

Table S1: Solar tracking success and limitations during the BB-FLUX deployment. During turns and turbulence as cause of unsuccessful tracking, the performance is further distinguished between GPS source.

\begin{tabular}{|l|l|l|l|l|}
\hline \multicolumn{2}{|l|}{ Description } & Frequency [\%] & MMQ (instrument) & OSDS (aircraft) \\
\hline--- & Sun tracking & 79.57 & -- & -- \\
\hline \multirow{2}{*}{ Mechanical } & Geometry & 6.48 & -- & -- \\
\cline { 2 - 5 } & Turns & 5.99 & 0.85 & 5.14 \\
\cline { 2 - 5 } & Motor & 1.54 & -- & - \\
\hline \multirow{2}{*}{ Atmospheric } & Turbulence & 0.97 & 0.18 & 0.79 \\
\cline { 2 - 6 } & Clouds \& other & $5.45 *$ & -- & -- \\
\hline
\end{tabular}

* $2.5 \%$ expected for no sun due to clouds based on flight notes and UWKA forward-facing camera images. 


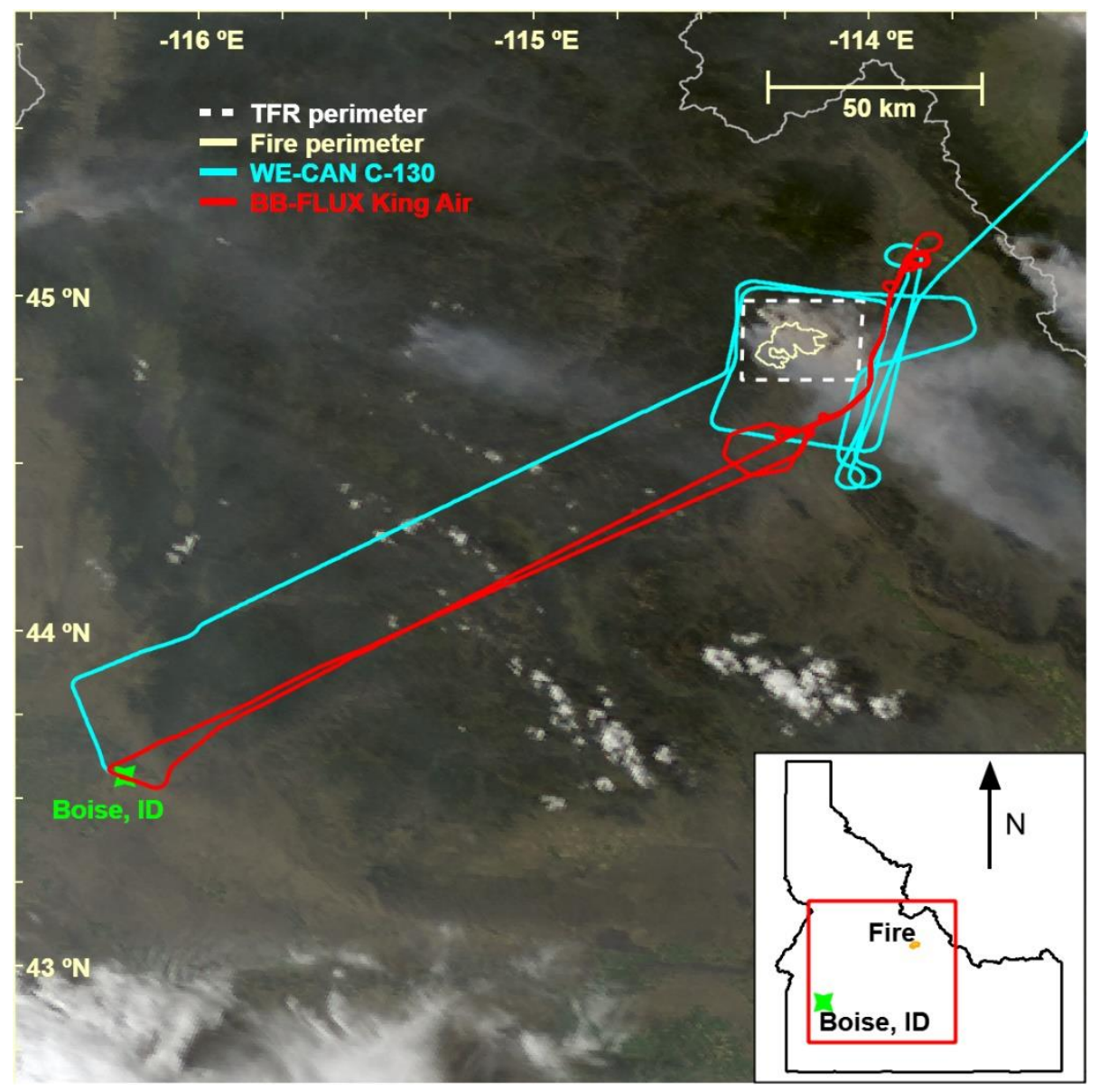

Figure S6. Research flight (RF) tracks of BB-FLUX RF13 and WE-CAN RF11 on 15 August 2018 to the Rabbit Foot Fire near Boise, Idaho. The fire perimeter and temporary flight restriction (TFR) zone are shown. Background image is from GOES-16 at 22:27 UTC. 


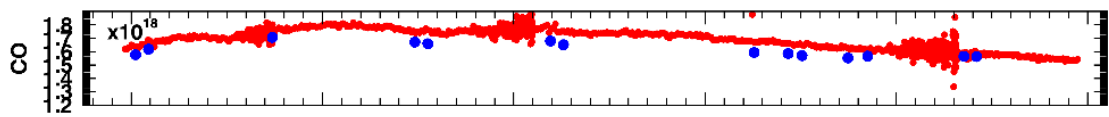

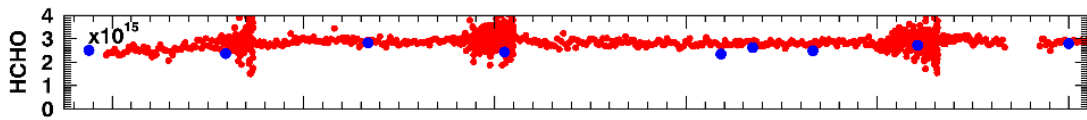
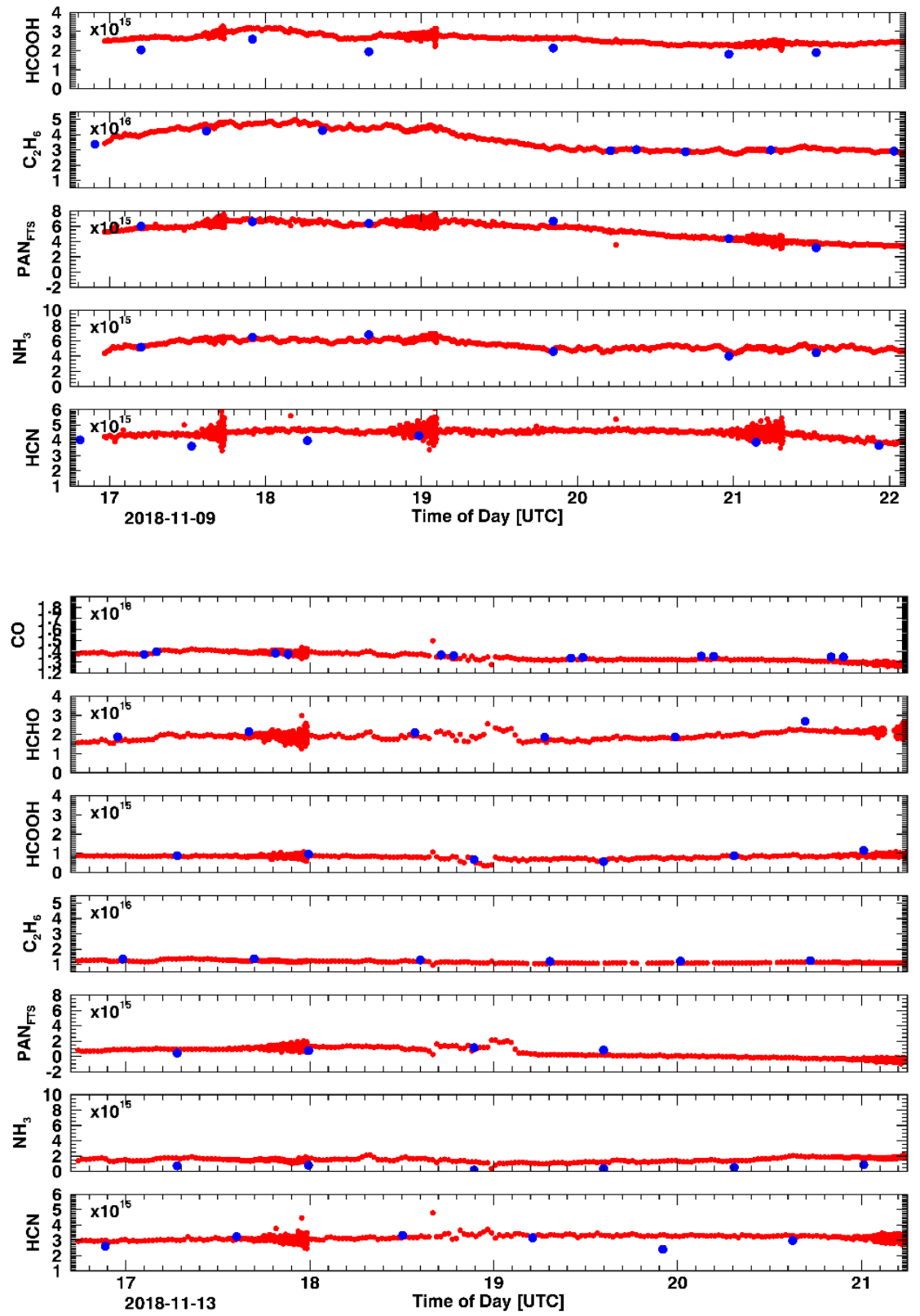

Figure S7. Co-located measurements of CU AirSOF at NCAR in Boulder, Colorado with the HR-NCAR-FTS. Blue: measurements of the HR-NCAR-FTS; red: individual CU AirSOF measurements (variable integration time). 
Table S2: Comparisons of median NEMR (Std. Dev./ Error due to the choice of background subtraction

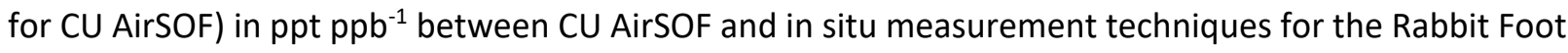
Fire, and comparison to those derived from commonly used EFs in models for temperate forests.

\begin{tabular}{|c|c|c|c|c|c|}
\hline Species & Measurement & $\begin{array}{l}\text { NEMR } \\
(<21: 45 \text { UTC) }\end{array}$ & $\begin{array}{l}\text { NEMR } \\
\text { Uncertainty }\end{array}$ & $\begin{array}{l}\text { NEMR } \\
(>21: 45 \text { UTC) }\end{array}$ & $\begin{array}{l}\text { NEMR from literature } \\
\text { (N=Number samples) }\end{array}$ \\
\hline \multirow[t]{2}{*}{$\mathrm{C}_{2} \mathrm{H}_{4}$} & SOF & $12.5(0.7 / 0.4)$ & $17 \%(2.1)$ & $16.9(0.6 / 0.3)$ & \multirow{2}{*}{$\begin{array}{ll}12.6(6.0)^{1} & (\mathrm{~N}=\mathrm{NA}) \\
9.8(5.0)^{2} & (\mathrm{~N}=21) \\
10.3(9.4)^{3} & (\mathrm{~N}=146)\end{array}$} \\
\hline & AWAS & 13.2 (1.1) & $11 \%(1.5)$ & |-- & \\
\hline \multirow[t]{2}{*}{$\mathrm{C}_{2} \mathrm{H}_{6}$} & SOF & $7.5(0.3 / 0.4)$ & $12 \%(0.9)$ & $8.5(0.4 / 0.1)$ & \multirow{2}{*}{$\begin{array}{ll}11.7(8.2)^{1} & (\mathrm{~N}=\mathrm{NA}) \\
5.7(5.3)^{2} & (\mathrm{~N}=21) \\
3.9(2.5)^{3} & (\mathrm{~N}=39)\end{array}$} \\
\hline & AWAS & $8.8(0.3)$ & $11 \%(1.0)$ & |-- & \\
\hline \multirow[t]{3}{*}{$\mathrm{HCHO}$} & SOF & $19.5(0.8 / 0.6)$ & $38 \%(7.4)$ & $24.4(0.3 / 0.0)$ & \multirow{3}{*}{$\begin{array}{ll}23.8(14.6)^{1} & (\mathrm{~N}=\mathrm{NA}) \\
16.8(9.4)^{2} & (\mathrm{~N}=16) \\
15.9(9.9)^{3} & (\mathrm{~N}=116)\end{array}$} \\
\hline & PTR-TOF-MS & 18.6 (1.3) & $21 \%$ (3.9) & --- & \\
\hline & TOGA & $21.4(2.1)$ & $57 \%(12.2)$ & -- & \\
\hline \multirow[t]{2}{*}{ PAN } & SOF & $4.1(0.2 / 0.5)$ & $16 \%(0.7)$ & $1.7(0.2 / 0.1)$ & \multirow[t]{2}{*}{$N A^{\wedge}$} \\
\hline & NCAR CIMS & $2.7(0.2)$ & $42 \%(1.1)$ & --- & \\
\hline \multirow[t]{2}{*}{$\mathrm{NH}_{3}$} & SOF & $16.5(0.4 / 1.2)$ & $11 \%(1.8)$ & $16.7(0.7 / 0.4)$ & \multirow{2}{*}{ 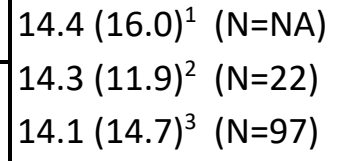 } \\
\hline & TILDAS & $14.8(1.9)$ & $17 \%(2.5)$ & |-- & \\
\hline \multirow[t]{5}{*}{$\mathrm{HCOOH}$} & SOF & $4.4(0.1 / 1.1)$ & $13 \%(0.6)$ & $2.9(0.3 / 0.4)$ & \multirow{5}{*}{$\begin{array}{l}2.4(2.4)^{1} \\
4.8(6.7)^{2} \\
1.9(1.8)^{3}\end{array}$} \\
\hline & $\mathrm{I}^{-\mathrm{CIMS}^{4}}$ & 7.3 (1.2) & $42 \%(3.1)$ & --- & \\
\hline & I-CIMS 5 & 10.1 (2.0) & $42 \%(4.2)$ & |-- & \\
\hline & PTR-TOF-MS 4 & $6.1(0.7)$ & $14 \%(0.9)$ & -- & \\
\hline & PTR-TOF-MS ${ }^{5}$ & $7.3(1.1)$ & $14 \%(1.0)$ & -- & \\
\hline \multirow[t]{3}{*}{$\mathrm{CH}_{3} \mathrm{OH}$} & SOF & $14.8(0.8 / 0.6)$ & $34 \%(5.0)$ & $13.8(0.0 / 0.2)$ & \multirow{3}{*}{ 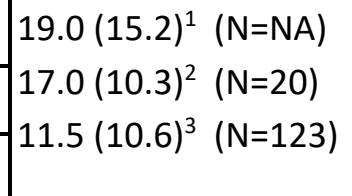 } \\
\hline & PTR-TOF-MS & 13.1 (1.2) & $14 \%(1.8)$ & --- & \\
\hline & TOGA & 16.1 (1.3) & $42 \%(6.8)$ & |-- & \\
\hline \multirow[t]{3}{*}{$\mathrm{HCN}$} & SOF & $2.6(0.2 / 0.4)$ & $43 \%(1.1)$ & $2.6(0.1 / 0.1)$ & \multirow{3}{*}{$\begin{array}{l}8.5(3.8)^{1} \\
5.9(4.4)^{2} \\
3.5(2.6)^{3}\end{array}$} \\
\hline & TOGA & $2.6(0.4)$ & $28 \%(0.7)$ & --- & \\
\hline & I-CIMS & $2.5(0.3)$ & $42 \%(1.1)$ & |-- & \\
\hline
\end{tabular}

${ }^{*}$ Given in percent and (absolute value in ppt $\mathrm{ppb}^{-1}$ )

${ }^{1}$ Akagi et al. ${ }^{1}$

${ }^{2}$ Andreae ${ }^{2}$

${ }^{3}$ Prichard et al. ${ }^{3}$

${ }^{4}$ Boundary layer background

${ }^{5}$ Standard background subtraction 
not available, considered with other VOCs

Table S3: Comparisons of individual calculated NEMR in ppt $\mathrm{ppb}^{-1}$ between CU AirSOF and in situ measurement techniques for the Rabbit Foot Fire with physical plume age between 0.5-1.5 $\mathrm{h}$.

\begin{tabular}{|c|c|c|c|c|c|c|c|c|c|}
\hline & & $20: 35$ & A & $B$ & C & D & $E$ & $\mathrm{~F}$ & $22: 39$ \\
\hline Species & Measurement & \multicolumn{8}{|c|}{ Individual Rabbit Foot Fire NEMRs } \\
\hline \multirow[t]{2}{*}{$\mathrm{C}_{2} \mathrm{H}_{4}$} & SOF & 12.5 & 12.4 & 12.3 & 11.3 & 12.7 & 13.6 & 17.3 & 16.5 \\
\hline & AWAS & --- & NA & 13.1 & 13.5 & 11.1 & 13.2 & --- & --- \\
\hline \multirow[t]{2}{*}{$\mathrm{C}_{2} \mathrm{H}_{6}$} & SOF & 7.8 & 7.5 & 7.5 & 7.4 & 7.2 & 8.0 & 8.2 & 8.8 \\
\hline & AWAS & --- & NA & 8.9 & 9.3 & 8.5 & 8.7 & --- & --- \\
\hline \multirow[t]{3}{*}{$\mathrm{HCHO}$} & SOF & 19.2 & 20.3 & 18.8 & 18.5 & 19.7 & 20.7 & 24.2 & 24.7 \\
\hline & PTR-TOF-MS & --- & 20.6 & 17.0 & 18.1 & 18.6 & 19.1 & --- & --- \\
\hline & TOGA & --- & 21.4 & 18.7 & 21.3 & 24.1 & 23.6 & --- & --- \\
\hline \multirow[t]{2}{*}{ PAN } & SOF & 4.2 & 4.2 & 3.8 & 4.0 & 4.1 & 3.9 & 1.8 & 1.5 \\
\hline & NCAR CIMS & --- & 2.7 & 2.6 & 3.0 & 2.7 & 2.6 & $\mid--$ & --- \\
\hline \multirow[t]{2}{*}{$\mathrm{NH}_{3}$} & SOF & 16.4 & 16.4 & 16.9 & 16.3 & 16.5 & 17.2 & 17.1 & 16.2 \\
\hline & TILDAS & --- & 11.9 & 16.0 & 14.0 & 16.8 & 14.8 & --- & |-- \\
\hline \multirow[t]{5}{*}{$\mathrm{HCOOH}$} & SOF & 4.5 & 4.4 & 4.3 & 4.5 & 4.3 & 4.1 & 3.1 & 2.6 \\
\hline & I-CIMS $^{1}$ & -- & 5.2 & 6.1 & 7.8 & 7.3 & 8.1 & -- & --- \\
\hline & I-CIMS ${ }^{2}$ & -- & 10.1 & 7.4 & 12.2 & 8.2 & 10.9 & -- & -- \\
\hline & PTR-TOF-MS ${ }^{1}$ & --- & 5.4 & 5.9 & 6.7 & 6.1 & 7.3 & & \\
\hline & PTR-TOF-MS² & -- & 7.3 & 6.7 & 9.2 & 6.7 & 8.5 & --- & --- \\
\hline \multirow[t]{3}{*}{$\mathrm{CH}_{3} \mathrm{OH}$} & SOF & 14.9 & 13.9 & 14.7 & 13.9 & 15.9 & 15.2 & 13.8 & 13.8 \\
\hline & PTR-TOF-MS & --- & 13.0 & 14.2 & 14.0 & 11.2 & 13.1 & --- & --- \\
\hline & TOGA & --- & 14.6 & 14.3 & 17.2 & 16.1 & 16.9 & --- & --- \\
\hline \multirow[t]{3}{*}{$\mathrm{HCN}$} & SOF & 2.6 & 2.7 & 3.0 & 2.6 & 2.5 & 2.5 & 2.6 & 2.5 \\
\hline & TOGA & --- & 2.4 & 2.6 & 2.6 & 3.3 & 3.0 & --- & --- \\
\hline & I-CIMS & --- & 2.1 & 2.5 & 2.5 & 2.7 & 2.7 & --- & --- \\
\hline
\end{tabular}

${ }^{1}$ Boundary layer background

${ }^{2}$ Standard background subtraction 


\section{References}

(1) Akagi, S. K.; Yokelson, R. J.; Wiedinmyer, C.; Alvarado, M. J.; Reid, J. S.; Karl, T.; Crounse, J. D.; Wennberg, P. O. Emission Factors for Open and Domestic Biomass Burning for Use in Atmospheric Models. Atmos. Chem. Phys. 2011, 11 (9), 4039-4072. https://doi.org/10.5194/ACP-11-40392011.

(2) Andreae, M. O. Emission of Trace Gases and Aerosols from Biomass Burning - An Updated Assessment. Atmos. Chem. Phys. 2019, 19 (13), 8523-8546. https://doi.org/10.5194/ACP-198523-2019.

(3) Prichard, S. J.; O’Neill, S. M.; Eagle, P.; Andreu, A. G.; Drye, B.; Dubowy, J.; Urbanski, S.; Strand, T. M.; Prichard, S. J.; O’Neill, S. M.; Eagle, P.; Andreu, A. G.; Drye, B.; Dubowy, J.; Urbanski, S.; Strand, T. M. Wildland Fire Emission Factors in North America: Synthesis of Existing Data, Measurement Needs and Management Applications. Int. J. Wildl. Fire 2020, 29 (2), 132-147.

https://doi.org/10.1071/WF19066. 